\title{
Interactive comment on "Evaluating Vehicle Emission Control Policies using on-Road Mobile Measurements and Continuous Wavelet Transform: a Case Study during the Asia-Pacific Economic Cooperation Forum, China 2014" by Ziqiang Tan et al.
}

Anonymous Referee \#2

Received and published: 11 July 2016

Review of Tan et al. Journal: Atmospheric Chemistry and Physics Manuscript \#: ACP2016-460 Title: "Evaluating Vehicle Emission Control Policies using on-Road Mobile Measurements and Continuous Wavelet Transform: a case study during the AsiaPacific Economic Cooperation Forum, China 2014.".

In this paper the authors describe a very interesting air quality monitoring campaign to measure air pollution changes in Beijing resulting from policy decisions driven by the Asia-Pacific Economic Cooperation (APEC) Forum, November 3-12, 2014. The goal 
of the study was to develop methods to quantify reductions of traffic emissions during APEC relative to before and after APEC. To do this, the authors made two trips per day (nighttime and daytime) for six, ten, and ten days before, during, and after APEC, respectively, in their mobile monitoring lab around the 4th ring road around Beijing and measured a suite of traffic- and combustion-related air pollutants. They then used continuous wavelet transformation to separate out traffic-related concentration spikes from their data and quantified emissions reductions attributable to APEC-related traffic restrictions. The authors argue that studies of this kind are needed to better define methods for separating traffic from non-traffic emissions of pollutants as well as to measure the effectiveness of air pollution control measures in cities. While the paper is very interesting and the data is quite unique and compelling, I cannot recommend the manuscript for publication in its current form. In addition to many relatively minor problems with the clarity of the data presentation and writing (indicated below), I found more significant problems having to do with contribution of the CWT method to the literature. On line 29-30 of the abstract, the authors write that the "CWT method represents on-road emissions better than other methods reported in the literature", but they offer no proof of why this is so.

\section{Detailed Comments:}

Major comments: 1 . It is not clear what this study adds to the literature on the subject. What critical questions does it answer, what is novel about the study design, methods, etc.? How do the results compare to previous studies? Without properly placing the results of the study in the context of the larger literature it is not possible for the reader (or reviewer) to judge its value.

2. Evaluation of the CWT approach for quantifying instantaneous emissions needs more emphasis. First, briefly describe what kinds of signals have been decomposed using CWT, and indicate the basis for why you think CWT is an appropriate tool for air pollution applications. If previous air pollution studies have used CWT then it would be sufficient to cite that literature. Next, describe how your results (\% reductions of 
traffic-related emissions during APEC) obtained using the CWT methods compare to results obtained with other widely reported methods. Finally, indicate how sensitive your \% reductions are to choice of signal processing method and comment on how do we know which method is better?

Other comments: 1 . The introduction is too long. The total length of the MS is 23 pages and the introduction is 5.5 pages. The introduction should be cut at least in half. It need only contain a clear statement of the problem, a short summary of the findings and shortcomings of earlier studies, a clear statement of the gaps in the literature and the study objectives to address the gaps.

2. A study limitation section is missing. This is a challenging study with many variables that could not be fully controlled. The limitations of the study design should be discussed.

3. Use of the term "instantaneous concentration" to describe exhaust plumes from individual vehicles is misleading. Some plumes can linger for several 10 s of seconds before dissipating - it all depends on source strength and atmospheric mixing conditions. I think the term "short-term concentration spikes" is more appropriate and suggest its use in place of instantaneous concentrations.

4. Why are $\mathrm{O} 3$ and $\mathrm{SO} 2$ part of the traffic analysis? $\mathrm{O} 3$ is a secondary pollutant, who's formation is contributed to by traffic emissions, and $\mathrm{SO} 2$ is not a traffic-related pollutant unless vehicle fuels in use in Beijing contains lots of sulfur (but I suspect it is mostly from coal). Inclusion of these two pollutants in the main analysis (Figs 3 and 4) and the methods needs to be justified. Otherwise, the results for these two pollutants should be moved to the supporting information section.

5. Line 171. Why is PNC not reported in the results section? Arguably, it is one of the best indicators of traffic-related air pollution of the pollutants you have measured. This should be described in the text. 
6. Line 289-90. It is not clear why $\mathrm{O} 3$ increases during APEC when instantaneous NOx concentrations decrease. The explanation provided needs greater detail and a reference so that reader can understand why a "NOx-controlled regime" could lead to greater $\mathrm{O} 3$ when the opposite would be expected.

7. Line 309. Need to explain why this ratio is useful and cite references. Also, indicate what threshold values to use to indicate coal combustion.

8. Line 313. References supporting this statement should follow "concentrations".

9. Lines 317 and 323 . Your box plots in fig 4 are not showing averages. The horizontal lines show medians. Please describe the medians not the averages when describing fig 4 results.

10. Line 355. Regarding sources of BC, aren't diesel vehicles in Beijing a significant source of $B C$ ? Many readers will assume this to be the case. Please provide references that specifically indicate otherwise or modify the statement.

11. Line 390. On-road vehicle numbers. Would be very interesting to see this. If possible present a time series before/during/after APEC of vehicles on 4th ring road distinguishing gasoline and diesel vehicles and night and day populations of each.

12. Line 422-423. "increase in diesel buses". This needs to be better described (e.g., how many buses per hour and on which days - weekdays vs. weekends - etc.?) and it should come much earlier as well as appear in Table 1.

13. Line 432. Suggestion is made that the decline of instantaneous BC is due to decline of diesel vehicle emission. This seems to contradict lines 354-55. This apparent contradiction should be addressed.

14. Lines 461-467. These sentences should be deleted as they don't add materially to the conclusions section.

Printer-friendly version

Minor comments: 1 . Line 50. Indicate what you mean by "the main pollutants". Better 
to be explicit.

2. Line 51. Instead of "the frequency of polluted days has also been high", it is better to be explicit and report quantitative measures like \# of days/y where one or more pollutants (specifically indicated) exceed indicated regulatory thresholds.

3. Line 83. Not clear what is meant by "inventories". Do you mean "emission factors"? Please clarify in the text.

4. Section 2.1. indicate monitoring frequency of each monitor, also indicate limits of detection and quality assurance measures taken to assure high quality data.

5. Line 174. Delete barometric pressure since you don't report the results.

6. Lines 239-40. Add percentages of time winds blew from each direction as well as specify what is meant by high wind speed.

7. Line 244. Change "diffusion" to "dispersion" or "mixing". Same thing in lines 251 and 298.

8. Line 245. Change "transport" to advection". Same thing in line 251.

9. Line 246. Describe in text what is meant by "temperature declined with time". Do you mean daily as expected or over the course of the study period.

10. Lines 258-268. These results should go in table and summary statements should be reported.

11. Lines 272-3. Not clear what "these values" refers to. please specifically indicate in the text - before, during or after APEC?

12. Line 279-80. This first sentence needs greater explanation. Not clear what "similar temporal trends" the authors are referring to.

13. Line 395. Change to "the day in the APEC period".

Editorial: 1. Line 45. Change to "lack of effective control policies" 
2. Line 65. Delete "the"

3. Line 66. Delete first use of "the"

4. Line 68. Delete "enormous"

5. Line 70. Add "However," before "because" and delete "the"

Interactive

6. Line 89. Delete "the"

comment

7. Line 94. Delete "the"

8. Line 95. Delete "parameters" and change aerosol to aerosols.

9. Line 96. Change to "characterized using mobile monitoring".

10. Line 97. Delete "research platform"

11. Line 163. O3 is missing from the list.

12. Line 167. Delete "sets of"

13. Line 180-1. Delete this sentence. You've already said this on lines 148-9.

14. Line 187. Change to "control policies, particularly on traffic-related air pollution,"

15. Line 189. Change to "For example, "following the 2008 Olympics," and delete "since the 2008. .." on 191-192.

16. Line 194. Indicate how many days of monitoring.

17. Line 232. Change "24" to "2"

Table 1. indicate whether coal-fired control is continued in the APEC period. Clarify what is meant by "Trucks were limited. . .24 PM". Make the language consistent with line 201, otherwise the reader will be confused as was the reviewer.

Table 2. line 686. Change "The percentage declines" to "Reductions" 
Figure 1. I cannot read the scale or the ortho photo credit.

Figure 3. would be helpful to swap dot colors so that night is solid black and day open white.

Figure 4. not clear what this represents. Are these box plots of all the data for both day and night trips on all days or just the daily averages? Please clarify. What is the value of the north, south, east, west data presentation? The box plots indicate little difference between the directions, and little discussion is given to these results, which leads the reader to wonder why it is presented in the first place. It would be more interesting and possibly more meaningful to present the box plots based on predominant wind direction during the 3 periods. In the figure caption it lists $\mathrm{SO} 2, \mathrm{O} 3$ as traffic-related air pollutants. Many readers will not consider them to be so. please justify or remove them from the figure. Indicate in the caption that the horizontal line is (median). The whiskers do not represent "the error bars" but rather the 5th and 95th percentiles. Please clarify what is meant by "averaged" in the caption.

Figure 6. indicate date Nox data was collected. Also, this figure should come earlier in the paper before description of \% reductions.

Please also note the supplement to this comment:

http://www.atmos-chem-phys-discuss.net/acp-2016-460/acp-2016-460-RC2-

supplement.pdf

Interactive comment on Atmos. Chem. Phys. Discuss., doi:10.5194/acp-2016-460, 2016. 